\title{
A Cost-Effectiveness Model for Adjunctive Smoked Cannabis in the Treatment of Chronic Neuropathic Pain
}

Griffin A. Tyree, ${ }^{1}$ Reith Sarkar, ${ }^{1}$ Brandon K. Bellows, ${ }^{2}$ Ronald J. Ellis, ${ }^{3-5}$ Joseph Hampton Atkinson, ${ }^{3,4}$ Thomas D. Marcotte, Mark S. Wallace, ${ }^{4,6}$ Igor Grant, ${ }^{3,4}$ Yuyan Shi, James D. Murphy, ${ }^{8}$ and David J. Grelotti ${ }^{3,4, *}$

\begin{abstract}
Background: A recent meta-analysis affirmed the benefit of medicinal cannabis for chronic neuropathic pain, a disabling and difficult-to-treat condition. As medicinal cannabis use is becoming increasingly prevalent among Americans, an exploration of its economic feasibility is warranted. We present this cost-effectiveness analysis of adjunctive cannabis pharmacotherapy for chronic peripheral neuropathy.

Materials and Methods: A published Markov model comparing conventional therapies for painful diabetic neuropathy was modified to include arms for augmenting first-line, second-line (if first-line failed), or third-line (if firstand second-line failed) therapies with smoked cannabis. Microsimulation of 1,000,000 patients compared the cost (2017 U.S. dollars) and effectiveness (quality-adjusted life years [QALYS]) of usual care with and without adjunctive cannabis using a composite of third-party and out-of-pocket costs. Model efficacy inputs for cannabis were adapted from clinical trial data. Adverse event rates were derived from a prospective study of cannabis for chronic noncancer pain and applied to probability inputs for conventional therapies. Cannabis cost was derived from retail market pricing. Parameter uncertainty was addressed with one-way and probabilistic sensitivity analysis.

Results: Adding cannabis to first-line therapy was incrementally less effective and costlier than adding cannabis to second-line and third-line therapies. Third-line adjunctive cannabis was subject to extended dominance, that is, the second-line strategy was more effective with a more favorable incremental cost-effectiveness ratio of \$48,594 per QALY gained, and therefore, third-line adjunctive cannabis was not as cost-effective. At a modest willingness-to-pay threshold of $\$ 100,000 / Q A L Y$ gained, second-line adjunctive cannabis was the strategy most likely to be cost-effective. Conclusion: As recently proposed willingness-to-pay thresholds for the United States health marketplace range from $\$ 110,000$ to $\$ 300,000$ per QALY, cannabis appears cost-effective when augmenting second-line treatment for painful neuropathy. Further research is warranted to explore the long-term benefit of smoked cannabis and standardization of its dosing for chronic neuropathic pain.
\end{abstract}

Keywords: cost-effectiveness; medical marijuana; painful neuropathy; diabetic neuropathy; HIV neuropathy

\section{Introduction}

A growing body of scientific literature demonstrates reproducible efficacy of cannabis in the treatment of several medical conditions, including chronic neuropathic pain. Clinical trials of oral, ${ }^{1-5}$ smoked, ${ }^{6-9}$ and vapor- ized $^{10,11}$ cannabis and cannabinoids have all demonstrated analgesic benefit of medicinal cannabis in the treatment of this costly ${ }^{12}$ and disabling ${ }^{13,14}$ condition. A recent meta-analysis of individual patient data from five randomized controlled trials of inhaled

\footnotetext{
'School of Medicine, University of California San Diego, La Jolla, California.

${ }^{2}$ Division of General Medicine, Columbia University, New York, New York.

${ }^{3}$ Department of Psychiatry, University of California San Diego, La Jolla, California.

${ }^{4}$ University of California Center for Medicinal Cannabis Research, San Diego, California.

Departments of ${ }^{5}$ Neurosciences, ${ }^{6}$ Anesthesiology, ${ }^{7}$ Family Medicine and Public Health, and ${ }^{8}$ Radiation Medicine and Applied Science, University of California San Diego, La Jolla, California.

*Address correspondence to: David J. Grelotti, MD, Center for Medicinal Cannabis Research, University of California San Diego, 220 Dickinson Street, Suite B, San Diego, CA 92103-8231, E-mail: dgrelotti@ucsd.edu
}

(c) Griffin A. Tyree et al. 2019; Published by Mary Ann Liebert, Inc. This Open Access article is distributed under the terms of the Creative Commons License (http://creativecommons.org/licenses/by/4.0), which permits unrestricted use, distribution, and reproduction in any medium, provided the original work is properly cited. 
cannabis demonstrated pain relief comparable to gabapentin. ${ }^{15}$ Treatment guidelines for neuropathic pain recommend consideration of cannabinoids as thirdline agents. ${ }^{16}$

An increasing number of patients are using cannabis for medical reasons, ${ }^{17-23}$ but how do we know if the health benefits gained with medicinal cannabis are worth the added cost? Cost-effectiveness analysis (CEA) compares the costs and health benefits of two or more interventions to determine their value. A treatment is considered cost-effective when the ratio of incremental costs to incremental health benefits, known as the incremental cost-effectiveness ratio (ICER), ${ }^{24}$ is less than a health care payer's willingness to pay for the health benefit. Quality-adjusted life years (QALYs), which incorporate both quality of life and longevity, are the recommended measure of health benefit. ${ }^{24,25}$

Conversely, treatment is considered dominatedand categorically not cost-effective-when it is less effective but more costly than an alternative, and extendedly dominated when there is another treatment alternative to usual care with a lower ICER value. In the United States, $\$ 50,000$ per QALY is a commonly accepted willingness-to-pay threshold, but expert opinion estimates that it likely ranges from $\$ 110,000$ to $\$ 300,000$ per QALY. ${ }^{26-28}$

To our knowledge, the only cost-effectiveness studies of cannabis or cannabinoids evaluated nabiximols and dronabinol for multiple sclerosis (MS), ${ }^{29}$ and no studies have assessed the cost-effectiveness of smoked medicinal cannabis for any condition. As federal regulations prohibit health plans from covering medicinal cannabis and patient expenses are out-of-pocket, knowing the cost-effectiveness of medicinal cannabis may impact how providers advise its use in patients suffering from chronic neuropathic pain.

As the majority of patients in placebo-controlled trials of medicinal cannabis were administered cannabis in addition to an existing pain regimen, ${ }^{6-8,11}$ there is sufficient data to support an exploratory study of the cost-effectiveness of smoked cannabis as adjunctive therapy in the treatment of chronic neuropathic pain. The purpose of this exploratory computer simulation study was to assess the cost-effectiveness of augmenting first-line, second-line, or third-line standard therapies for neuropathic pain with smoked cannabis in treatment-naive patients over 1 year from a U.S. health care sector perspective. We also sought to assess the robustness of our simulation to changes in parameter inputs and assumptions. ${ }^{30}$

\section{Materials and Methods}

\section{Overview}

CEAs often use branching decision models constructed in specialized software to compare interventions in simulated patients. In CEA models, simulated patients experience clinical outcomes related to health state utility and decrements (e.g., clinical improvement, intolerable side effects, or death) and incur costs (e.g., prescription medication fills, physician office visits, or hospitalizations) based on input probabilities, which are commonly derived from published literature. ${ }^{30}$

For this analysis, we constructed a CEA model by adding adjunctive cannabis to the treatment arms of a published microsimulation (i.e., individual-patient simulation) model of painful diabetic peripheral neuropathy (pDPN). ${ }^{31}$ While the efficacy of smoked cannabis from clinical trials represents a heterogeneous group of conditions causing neuropathic pain, we did not identify a published microsimulation model for chronic neuropathic pain due to mixed etiologies. Although multiple published CEAs of chronic neuropathic pain due to a single etiology were identified, ${ }^{31-34}$ the model by Bellows et al. best approximated clinical practice by allowing patients to switch between standard therapy agents when they experienced poor pain relief or adverse events. ${ }^{31}$

We therefore adapted this model to estimate the costs (2017 U.S. dollars), QALYs, and cost-effectiveness of augmenting standard therapy agents for neuropathic pain with smoked cannabis by adding parameters to simulate adjunctive cannabis use.

\section{Model structure}

We simulated the cost and QALY outcomes of $1,000,000$ treatment-naive patients newly diagnosed with neuropathic pain. Following Bellows et al., baseline age and pain score were assigned from normal distributions derived from pooled, weighted means and standard deviations from clinical trials of four standard therapy agents for neuropathic pain (i.e., desipramine, duloxetine, gabapentin, and pregabalin). ${ }^{31}$ As the previous analysis found that initiating duloxetine in treatment-naive patients with pDPN was the most cost-effective first-line therapy, ${ }^{31}$ we assumed that each patient would receive standard therapy beginning with duloxetine.

In the event of drug failure, patients then switched randomly to one of three remaining standard therapy agents. In our model, adjunctive cannabis was initiated according to four treatment strategies: (1) never (the "usual care" arm), (2) at the start of treatment (first- 
line adjunctive cannabis), (3) after failing one standard therapy agent (second-line adjunctive cannabis), or (4) after failing two standard therapy agents (third-line adjunctive cannabis).

All patients began the simulation in the moderateto-severe pain state (i.e., score $\geq 4$ on an 11-point Likert scale). Patients were assessed stepwise for mortality, adherence, adverse events, and pain relief following procedures developed by Bellows et al. ${ }^{31}$ modified to a 6-week cycle to account for additional pain relief and/or adverse events related to adjunctive cannabis use (Fig. 1). Six-week cycle length was selected as this represents a patient with moderate-to-severe pain whose treatment is actively being optimized.

At the end of each 6-week cycle, patients with a pain score $<4$ were assumed to have good pain relief and improved quality of life. If so, patients would remain on that treatment. Patients with a pain score $\geq 4$ had poor pain relief and decreased quality of life. If patients had two or more 6-week cycles of inadequate pain control or experienced intolerable or serious adverse events (SAEs) in any one cycle, it was assumed that they would switch drugs. Patients who were nonadherent to adjunctive cannabis experienced poor pain relief through two cycles of adjunctive cannabis treatment, or experienced intolerable or SAEs while taking adjunctive cannabis, were considered to have failed cannabis and could not restart the drug.

\section{Model inputs}

Parameters selected for the model, model inputs, and their distribution types are presented in Table 1. Model inputs for the efficacy and adverse event rates of standard therapy agents were extracted from Bellows et al. ${ }^{31}$ Costs were assessed from a U.S. health care sector perspective, which incorporates both third-party payer direct medical costs (i.e., what insurers pay) and patient out-of-pocket costs, to account for both health plancovered prescription medications and medicinal cannabis, which patients must pay for themselves. ${ }^{24}$ The cost of standard therapy agents, health state utility, and utility decrements due to adverse events were adapted from Bellows et al. (SupplementaryTable S1). ${ }^{31}$

To derive parameters for efficacy of smoked cannabis, we examined clinical trials of whole-plant cannabis in adults with chronic peripheral neuropathic pain if the study drug was administered in cigarette form, 24$h$ average pain scores were reported, pain reduction was

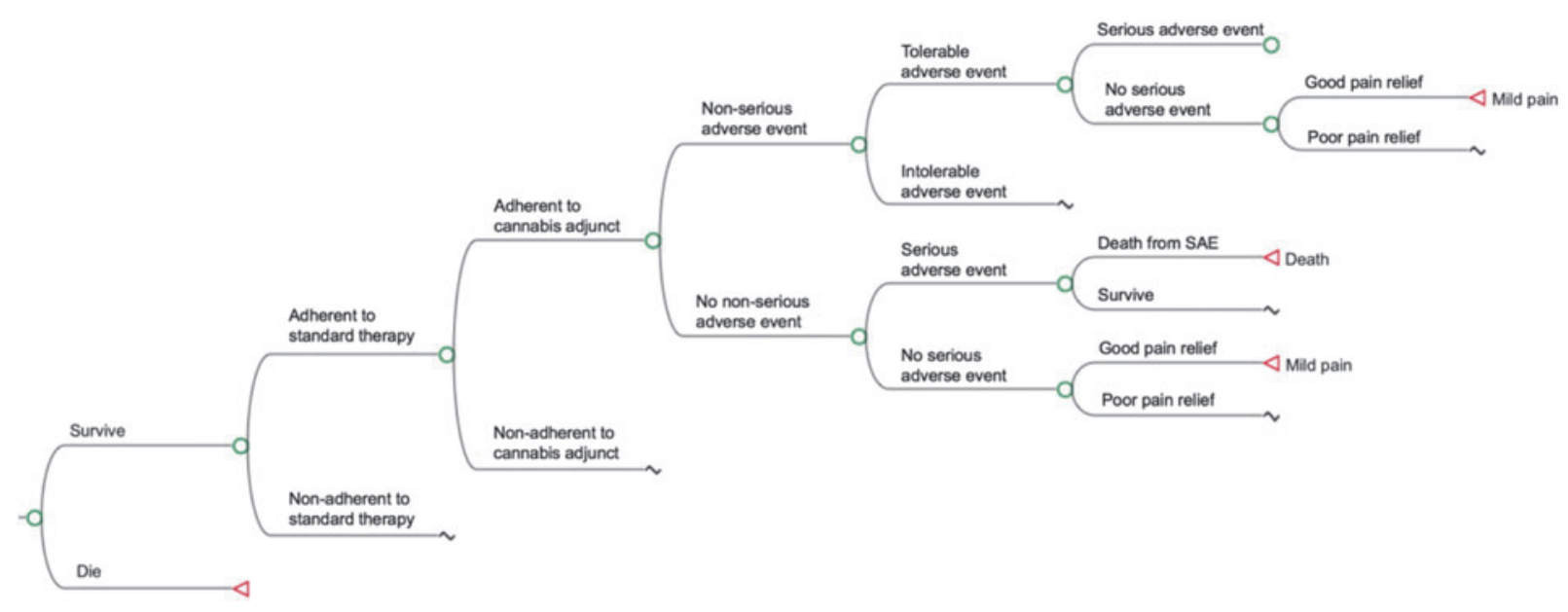

FIG. 1. Abbreviated model overview. Pictured is a node structure wherein adjunctive cannabis is integrated into a treatment model using standard therapy agents described by Bellows et al. ${ }^{31}$ Beginning in a moderateto-severe pain health state, simulated patients are assessed stepwise for mortality, adherence, tolerable or intolerable adverse events, $\mathrm{SAE}$, and quality of pain relief. Patients who die are removed from the simulation and do not transition further. Nonadherence disqualifies a patient from experiencing either pain relief or adverse events from a given agent. Serious or intolerable adverse events trigger discontinuation of current therapy (with or without adjunctive cannabis) and drug-switching. Patients who attain good pain relief (pain score $<4$ ) transition to the mild pain state at the end of the cycle. In the absence of good pain relief, patients remain in moderate-to-severe pain at the end of the cycle. SAE, serious adverse events. 
Table 1. Parameter Distribution Inputs

\begin{tabular}{|c|c|c|}
\hline Parameter & Model inputs & $\begin{array}{l}\text { Distribution } \\
\text { type }\end{array}$ \\
\hline \multicolumn{3}{|l|}{ Baseline values, ${ }^{31}$ mean (SD) } \\
\hline Age & $59.72(9.79)$ & Normal \\
\hline Pain score & $6.20(1.52)$ & Normal \\
\hline \multicolumn{3}{|c|}{ Pain score reduction, ${ }^{31}$ mean (SD) } \\
\hline Duloxetine & $2.57(2.31)$ & Normal \\
\hline Desipramine & $1.99(2.16)$ & Normal \\
\hline Gabapentin & $2.42(2.34)$ & Normal \\
\hline Pregabalin $^{31}$ & $2.59(1.87)$ & Normal \\
\hline Cannabis $^{35}$ & $1.11(2.38)$ & Normal \\
\hline \multicolumn{3}{|c|}{ Probability of nonserious AEs, ${ }^{31}$ proportion (SE) } \\
\hline Duloxetine & $66.0 \%(1.2 \%)$ & Beta \\
\hline Desipramine & $74.4 \%(4.9 \%)$ & Beta \\
\hline Gabapentin & $66.4 \%(2.5 \%)$ & Beta \\
\hline Pregabalin & $69.1 \%(1.5 \%)$ & Beta \\
\hline Cannabis $^{35, a}$ & $58.6 \%(3.4 \%)$ & Beta \\
\hline \multicolumn{3}{|c|}{ Probability of intolerable $\mathrm{AEs}{ }^{31}$ proportion (SE) } \\
\hline Duloxetine & $15.7 \%(1.2 \%)$ & Beta \\
\hline Desipramine & $13.8 \%(4.5 \%)$ & Beta \\
\hline Gabapentin & $14.7 \%(2.3 \%)$ & Beta \\
\hline Pregabalin & $12.5 \%(1.3 \%)$ & Beta \\
\hline Cannabis ${ }^{35, a}$ & $4.6 \%(1.4 \%)$ & Beta \\
\hline \multicolumn{3}{|c|}{ Probability of serious $\mathrm{AEs}^{31}$ proportion (SE) } \\
\hline Duloxetine & $2.4 \%(0.4 \%)$ & Beta \\
\hline Desipramine & $1.3 \%(1.3 \%)$ & Beta \\
\hline Gabapentin & $4.0 \%(1.1 \%)$ & Beta \\
\hline Pregabalin & $2.6 \%(0.5 \%)$ & Beta \\
\hline Cannabis ${ }^{35, a}$ & $0.5 \%(0.5 \%)$ & Beta \\
\hline \multicolumn{3}{|c|}{ Cannabis AE risk modifier, ${ }^{35}$ odds ratio $(95 \% \mathrm{Cl})$} \\
\hline Nonserious AEs & $1.74\left(1.42-2.14^{\mathrm{b}}\right)$ & Logistic \\
\hline $\begin{array}{l}\text { Nonserious AEs-no } \\
\text { active use }\end{array}$ & $2.07\left(1.59-2.70^{\mathrm{b}}\right)$ & Logistic \\
\hline Serious AEs & $1.08\left(0.57-2.04^{b}\right)$ & Logistic \\
\hline $\begin{array}{l}\text { Serious AEs-no } \\
\text { active use }\end{array}$ & $1.77\left(0.72-4.32^{\mathrm{b}}\right)$ & Logistic \\
\hline \multicolumn{3}{|c|}{ Risk of death from SAE ${ }^{59}$ (by age, years), proportion (SE) } \\
\hline $18-44$ & $1.2 \%(0.1 \%)$ & Beta \\
\hline $45-64$ & $1.6 \%(0.2 \%)$ & Beta \\
\hline $65-84$ & $1.9 \%(0.2 \%)$ & Beta \\
\hline$\geq 85$ & $2.6 \%(0.6 \%)$ & Beta \\
\hline \multicolumn{3}{|l|}{ Adherence, ${ }^{60}$ mean (SD) } \\
\hline Duloxetine & $0.86(0.18)$ & Beta \\
\hline Desipramine & $0.76(0.24)$ & Beta \\
\hline Gabapentin & $0.74(0.24)$ & Beta \\
\hline Pregabalin & $0.69(0.25)$ & Beta \\
\hline Cannabis $^{36}$ & 0.84 (95\% Cl: $\left.0.78-0.90^{\mathrm{b}}\right)$ & Beta \\
\hline $\begin{array}{l}\text { Adherence threshold } \\
\text { (assumed) }\end{array}$ & 0.8 (range: $0.5-1.0$ ) & Triangular \\
\hline \multicolumn{3}{|c|}{ Discontinuation rate, ${ }^{31}$ proportion (SE) } \\
\hline Duloxetine & $1.7 \%(0.4 \%)$ & Beta \\
\hline Desipramine & $2.6 \%(1.8 \%)$ & Beta \\
\hline Gabapentin & $2.3 \%(0.8 \%)$ & Beta \\
\hline Pregabalin & $3.9 \%(0.7 \%)$ & Beta \\
\hline Cannabis $^{35}$ & $10.7 \%(2.1 \%)$ & Beta \\
\hline \multicolumn{3}{|c|}{ Health state utilities, ${ }^{13}$ mean (SD) } \\
\hline Mild pain & $0.7(0.2)$ & Beta \\
\hline Moderate-to-severe pain & $0.39(0.33)$ & Beta \\
\hline \multicolumn{3}{|l|}{ Utility decrements, mean } \\
\hline Tolerable $A E^{61,62}$ & $0.05^{\mathrm{d}}$ & Beta \\
\hline Intolerable $A E^{63-65}$ & $0.11^{\mathrm{d}}$ & Beta \\
\hline Serious $A E^{64,66,67}$ & $0.12^{\mathrm{d}}$ & Beta \\
\hline \multicolumn{3}{|l|}{ Office visit costs, ${ }^{68}$ mean (SD) } \\
\hline Regular visit & $\$ 111(\$ 7)$ & Gamma \\
\hline SAE visit & $\$ 150(\$ 10)$ & Gamma \\
\hline & $\$ 51(\$ 4)$ & Gamma \\
\hline
\end{tabular}

Table 1. (Continued)

\begin{tabular}{|c|c|c|}
\hline Parameter & Model inputs & $\begin{array}{l}\text { Distribution } \\
\text { type }\end{array}$ \\
\hline \multicolumn{3}{|l|}{$\begin{array}{l}\text { Regular visit, out- } \\
\text { of-pocket }^{69}\end{array}$} \\
\hline SAE visit, out-of-pocket ${ }^{69}$ & $\$ 57(\$ 8)$ & Gamma \\
\hline \multicolumn{3}{|c|}{ SAE hospitalization costs ${ }^{59}$ (by age, years), mean (SE) } \\
\hline $18-44$ & $\$ 7,387(\$ 130)$ & Gamma \\
\hline $45-64$ & $\$ 9,447(\$ 165)$ & Gamma \\
\hline $65-84$ & $\$ 9,664(\$ 292)$ & Gamma \\
\hline$\geq 85$ & $\$ 8,658(\$ 340)$ & Gamma \\
\hline $\begin{array}{l}\text { Hospitalization out-of- } \\
\text { pocket costs }\end{array}$ & $\$ 70(\$ 37)$ & Gamma \\
\hline \multicolumn{3}{|c|}{ Standard therapy wholesale costs ${ }^{31,71}$ (1 month supply), mean (SD) } \\
\hline Duloxetine & $\$ 254(\$ 20)$ & Gamma \\
\hline Desipramine & $\$ 236(\$ 58)$ & Gamma \\
\hline Gabapentin & $\$ 305(\$ 99)$ & Gamma \\
\hline Pregabalin & $\$ 485(\$ 33)$ & Gamma \\
\hline \multicolumn{3}{|c|}{ Standard therapy out-of-pocket costs, ${ }^{11,72}$ mean (SE) } \\
\hline Duloxetine & $\$ 13.00(\$ 2.34)$ & Gamma \\
\hline Desipramine & $\$ 22.25(\$ 7.04)$ & Gamma \\
\hline Gabapentin & $\$ 8.79(\$ 3.54)$ & Gamma \\
\hline Pregabalin & $\$ 19.63(\$ 9.98)$ & Gamma \\
\hline \multicolumn{3}{|l|}{ Cannabis cost, mean (SD) } \\
\hline Price per gram $^{37}$ & $\$ 11.06(\$ 3.78)$ & Gamma \\
\hline \multicolumn{3}{|l|}{ Cannabis quantification ${ }^{6}$} \\
\hline Daily grams THC & $0.067(0.034)$ & Gamma \\
\hline Cannabis wastage & $38.9 \%(13.2 \%)$ & Beta \\
\hline
\end{tabular}

aFor cannabis "monotherapy," when patient is nonadherent to conventional agent but adherent to cannabis.

${ }^{\mathrm{b}}$ Distribution SD estimated as $1 / 4$ of $95 \% \mathrm{Cl}$.

${ }^{c}$ Range and distribution for adherence threshold used in probabilistic sensitivity analysis only.

${ }^{\mathrm{d}}$ Distribution SD estimated as $1 / 2$ of mean value.

$\mathrm{AE}$, adverse event; $\mathrm{Cl}$, confidence interval; $\mathrm{SAE}$, serious adverse event; $\mathrm{SD}$, standard deviation; $\mathrm{SE}$, standard error; THC, tetrahydrocannabinol.

reported on a numeric or Likert scale, duration was $\geq 5$ days, and results were published in English. We considered all etiologies for neuropathic pain. Two trials in HIV-associated sensory neuropathy were identified which met our criteria. ${ }^{6,7}$ However, we required access to study data to extract mean pain score reductions. Only Ellis et al. provided data for their study. Data from the other trial were not easily accessible (Donald Abrams, personal communication).

We converted the pain score reduction measures from a 100-point visual analog scale to an 11-point Likert scale to align with the parameters for standard therapy agents. We modeled the efficacy of adjunctive cannabis using the mean difference in pain score reductions between active cannabis and placebo cigarettes.

To model the change in probability of adverse events when cannabis is used to augment standard therapy, we applied a modifier to adverse event rates for standard therapy agents. This modifier was derived from adjusted odds ratios (ORs) for non-SAE and SAE calculated in the Cannabis for the Management of Pain: 
Assessment of Safety Study (COMPASS), ${ }^{35}$ which compared safety outcomes between chronic pain patients who did and did not self-treat with cannabis over a 1-year timeframe. When patients were nonadherent to standard therapy but adherent to cannabis, adverse event rates were derived from the proportion of participants in COMPASS who were exposed to cannabis and experienced adverse events that investigators considered related to the drug.

Dosing of cannabis was based on the administration schedule used by Ellis et al., that is, four times daily. ${ }^{6}$ Literature on adherence to medicinal cannabis is sparse, and no published study reports cannabis adherence as a scalar variable. Therefore, we estimated adherence to smoking cannabis four times daily as adherence to a four times daily-dosed medication in chronic disease, reported in a recent meta-analysis. ${ }^{36}$

We derived the cost per gram of cannabis flower from a study of transactions in the Washington state legal marketplace from 2014 to $2016 .{ }^{37}$ We modeled a cannabis whole-plant product containing $12.5 \%$ tetrahydrocannabinol (THC), which emulates the study cannabis used in COMPASS. ${ }^{35}$ The daily dose of THC was derived from the average daily dose delivered to participants by Ellis et al. in their clinical trial, ${ }^{6}$ a dose individually titrated to balance pain relief and tolerability.

\section{Analysis}

Effectiveness of each treatment strategy was expressed in QALYs. In the model, QALYs for each 6-week cycle were calculated by multiplying the utility of a patient's health state, determined by pain relief and adverse events, by the time spent in that health state. ${ }^{30}$ Utility, a measure of quality of life assigns a value between 0 (death) and 1 (perfect health) to represent severity of disability in a health state. Costs were also assessed on a 6-week cycle. Costs included both third-party and out-of-pocket costs due to the standard therapy agents, costs of an office visit to a physician or hospitalization, and, where applicable, costs of adjunctive cannabis. The cost-effectiveness of treatment arms was expressed using ICERs. We adjusted costs and QALYs at a rate $3 \%$ annually to account for inflation and adjusted future utility gains to their value at present. $^{24}$ All analyses were performed using TreeAge Pro 2018 (TreeAge Software, Inc., Williamstown, MA).

Base-case analysis. The goals of our analysis were twofold. First, we evaluated whether any adjunctive cannabis strategy was cost-effective compared to usual care by determining if the calculated ICER was at or below a willingness-to-pay threshold of $\$ 100,000$ per QALY. Second, we compared the relative cost-effectiveness of first-line, second-line, and third-line adjunctive cannabis to determine the strategy associated with the greatest value.

Sensitivity analysis. To assess the robustness of our findings to variations in model parameters-as some parameters may fluctuate in clinical practice-we performed both one-way and probabilistic sensitivity analyses. This is especially relevant to medicinal cannabis for which prices fluctuate widely by region and retailer and for which there remains uncertainty regarding long-term efficacy and rates of adverse events. ${ }^{37-40}$

In one-way sensitivity analysis, the model is run for multiple iterations and the mean of a single parameter is varied over a specified range. For cannabis adverse event rate modifiers and adherence to adjunctive cannabis, we defined this range as the $95 \%$ confidence interval of reported ORs. Mild pain health state utility, cannabis price per gram, daily THC dose, and adherence to standard therapy agents were all varied over \pm one standard deviation from the mean. Adherence threshold was varied from 0.5 to 1.0 (i.e., $50 \%$ adherence to $100 \%$ adherence). Non-SAE probabilities were varied over $\pm 25 \%$ from the mean. All other parameters were varied over $\pm 50 \%$ from the mean value.

We also performed probabilistic sensitivity analysis to account for uncertainty across all parameters at once. The model was run 10,000 times with new parameter values selected at each iteration from the model distributions used in base-case analysis (Table 1). While adherence threshold was static in base-case analysis, in probabilistic sensitivity analysis, it was sampled from a triangular distribution from 0.5 to 1.0. The resulting QALYs and costs were used to determine the relative cost-effectiveness of each treatment plan. The proportion of model iterations, in which a given treatment plan was most cost-effective compared to all other treatment arms, was plotted against a range of willingness-to-play thresholds, the cost-per-QALY value up to which interventions are considered cost-effective in a given context.

Alternate time horizons. In base-case, our model was analyzed with a 1-year time horizon. This was a function of limited data in the literature: the longest timeframe, in which adverse events were followed for users of a medicinal cannabis regimen, was 1 year. ${ }^{35}$ 
As use of an analgesic regimen beyond 1 year is more reflective of clinical practice for a chronic condition such as painful neuropathy, we examined the model with extended time horizons of 5 and 10 years. However, these estimates were highly exploratory as data on the efficacy and adverse effects of medicinal cannabis use in that timeframe are unavailable.

Alternate adverse event modifiers. Adverse event rate modifiers for cannabis-containing regimens were derived from adjusted ORs calculated in an observational cohort study, in which $65.6 \%$ of participants in the cannabis-exposed group were current users at baseline. ${ }^{35}$ Tolerance to adverse effects has been observed after repeated dosing. ${ }^{41}$ ORs for all adverse events and SAEs were higher when active cannabis users were excluded from analysis. We conducted a subanalysis using these ORs to simulate starting medicinal cannabis in a population who are not active users.

Cannabis wastage. The base-case model assumes that patients use cannabis with a high degree of efficiency, consuming only the amount corresponding to their prescribed dose of THC. To simulate loss of cannabis to waste (and the need to purchase a somewhat larger quantity of the drug than in base-case), we calculated a cannabis wastage term using supplemental data provided by Ellis et al. ${ }^{6}$ - defined as the proportion of unused cannabis cigarette at the end of a smoking session multiplied by THC concentration-and applied it to our model.

\section{Results}

Base-case analysis

The results of the base-case analysis are presented in Table 2. In the base-case analysis, usual care had the lowest mean cost $(\$ 6,397)$ per patient, followed by third-line $(\$ 6,641)$, second-line $(\$ 7,007)$, and first-line adjunctive cannabis strategies $(\$ 7,234)$. Second-line ad- junctive cannabis provided the greatest average QALYs per patient (0.489), followed by first-line $(0.488)$ and third-line (0.480) adjunctive cannabis, then usual care (0.476).

As it costs more while being less effective than second-line adjunctive cannabis, first-line adjunctive cannabis was dominated and not considered further. While both second- and third-line adjunctive cannabis strategies were more effective than usual care, secondline adjunctive cannabis yielded more QALYs and had a more favorable ICER versus usual care; thus, thirdline adjunctive cannabis was extendedly dominated and not considered further. Second-line adjunctive cannabis was cost-effective compared to usual care, with an ICER of $\$ 48,594$ per QALY gained.

\section{Sensitivity analyses}

As second-line adjunctive cannabis dominated or extendedly dominated both first-line and third-line strategies in base-case analysis, we structured one-way sensitivity analyses comparing second-line cannabis with usual care (Fig. 2). Our model was most sensitive to changes in adherence threshold, mild pain state utility, and moderate-to-severe pain state utility. The model was also sensitive-to a lesser extent-to changes in cannabis adherence and daily THC dose. For example, when cannabis adherence was reduced from $84 \%$ in base-case to $78 \%$, the ICER for secondline adjunctive cannabis tripled to $\$ 145,292$ per QALY. When daily THC dose required was raised from 0.067 to $0.101 \mathrm{~g}$ per day, the ICER for secondline cannabis increased to $\$ 68,220$ per QALY.

In probabilistic sensitivity analysis, usual care was most likely to be cost-effective up to a willingness-topay threshold of $\$ 60,000$ per QALY (Fig. 3). Above $\$ 60,000$ per QALY second-line adjunctive cannabis was the most cost-effective strategy, with a $62 \%$ probability of being the most cost-effective strategy at a willingness-to-pay threshold of \$100,000 per QALY.

Table 2. Average Cost and Efficacy of Base-Case Analysis

\begin{tabular}{|c|c|c|c|c|c|}
\hline \multirow[b]{2}{*}{ Treatment strategy } & \multicolumn{2}{|c|}{ Average } & \multicolumn{2}{|c|}{ Incremental } & \multirow[b]{2}{*}{ ICER (\$/QALY gained) } \\
\hline & Cost (\$U.S.) & Efficacy (QALY) & Cost & Efficacy & \\
\hline Usual care & $\$ 6,397$ & 0.476 & REF & REF & REF \\
\hline First-line adjunctive cannabis & $\$ 7,234$ & 0.488 & - & - & Dominated \\
\hline Second-line adjunctive cannabis & $\$ 7,007$ & 0.489 & $\$ 610$ & 0.013 & $\$ 48,594$ \\
\hline Third-line adjunctive cannabis & $\$ 6,641$ & 0.480 & - & - & Ext. dominated \\
\hline
\end{tabular}

All costs are in 2017 U.S. dollars. ICERs are calculated referent to the next least costly nondominated treatment option.

ICER, incremental cost-effectiveness ratio; REF, reference value; Ext. dominated, extendedly dominated; QALY, quality-adjusted life-years. 


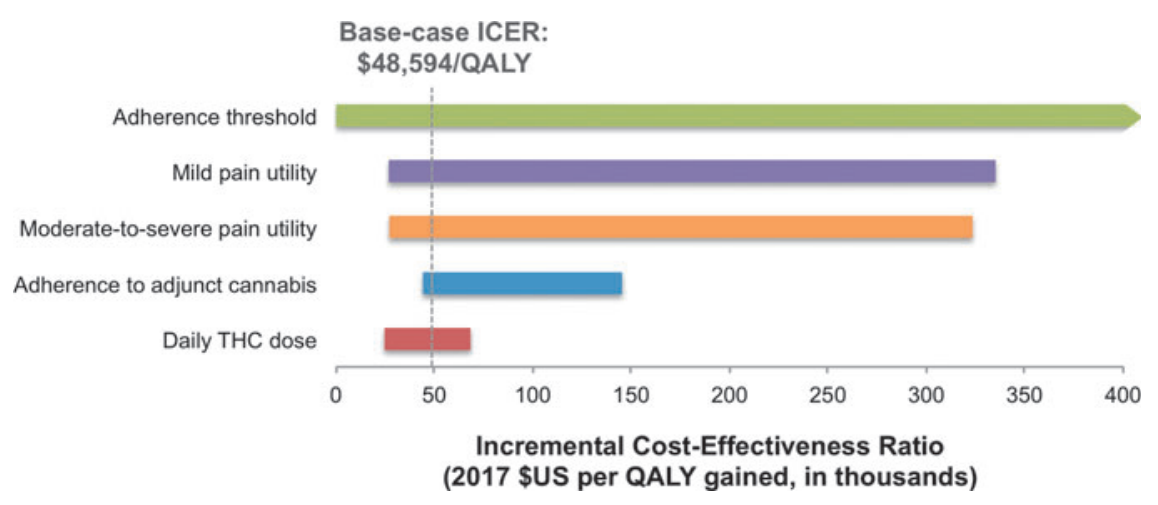

FIG. 2. One-way sensitivity analysis tornado diagram. ICER represents the incremental cost per QALY gained from second-line adjunctive cannabis when compared to usual care. The dotted vertical line represents the base-case ICER of $\$ 48,594 /$ QALY, while the horizontal bars indicate the magnitude of change in ICER caused by varying the parameter over its specified range. A negative ICER value, at which second-line adjunctive cannabis was dominated by usual care, is represented by an arrow tip on the end of the horizontal bar. All variables were examined in analysis; the five parameters shown caused the greatest change in ICER. Varying adherence threshold caused usual care to dominate second-line adjunctive cannabis. Varying moderate-to-severe pain state utility, mild pain state utility, and adherence to adjunctive cannabis over their respective ranges for sensitivity analysis caused the ICER to cross the $\$ 100,000 / Q A L Y$ threshold and second-line adjunctive cannabis to lose cost-effectiveness. However, second-line adjunctive cannabis remained cost-effective across the range of values for daily THC dose inputs. ICER, incremental cost-effectiveness ratio; QALY, quality-adjusted life-year; THC, tetrahydrocannabinol.

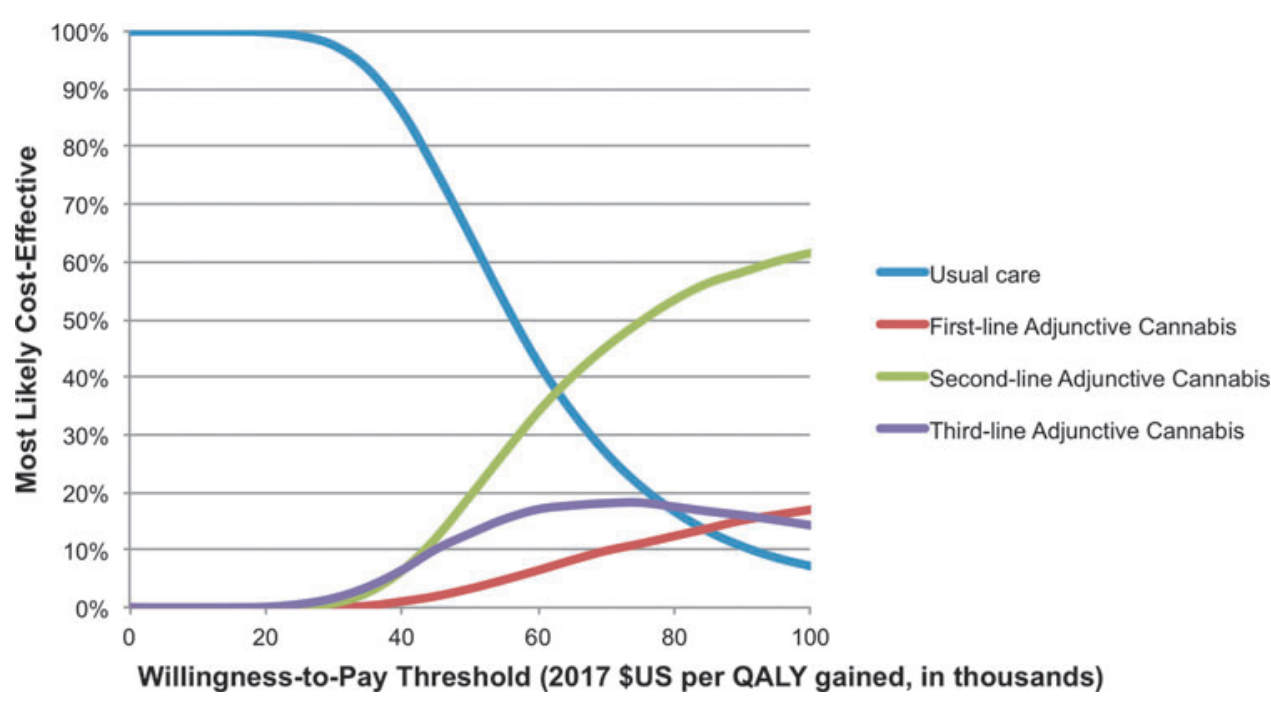

FIG. 3. Cost-effectiveness acceptability curve from probabilistic sensitivity analysis. Percentage of iterations, in which a given treatment strategy is most cost-effective, was plotted against escalating willingness-to-pay thresholds. In our model, cannabis-containing strategies were most cost-effective at willingness-to-pay thresholds of approximately $\$ 60,000$ per QALY gained and above. 
Table 3. Outcomes of Alternate Time Horizon, Adverse Event Rate, and Cannabis Wastage Analyses

\begin{tabular}{|c|c|c|c|c|}
\hline & Usual care & $\begin{array}{c}\text { First-line } \\
\text { adjunctive } \\
\text { cannabis }\end{array}$ & $\begin{array}{l}\text { Second-line } \\
\text { adjunctive } \\
\text { cannabis }\end{array}$ & $\begin{array}{l}\text { Third-line } \\
\text { adjunctive } \\
\text { cannabis }\end{array}$ \\
\hline \multicolumn{5}{|c|}{ Five-year time horizon } \\
\hline Average QALYs & 2.024 & 2.057 & 2.098 & 2.088 \\
\hline Average cost & $\$ 27,505$ & $\$ 29,035$ & $\$ 29,327$ & $\$ 28,865$ \\
\hline ICER & REF & Dominated & $\$ 45,968$ & $\$ 21,143$ \\
\hline \multicolumn{5}{|c|}{ Ten-year time horizon } \\
\hline Average QALYs & 3.469 & 3.507 & 3.563 & 3.557 \\
\hline Average cost & $\$ 47,937$ & $\$ 49,926$ & $\$ 50,333$ & $\$ 49,853$ \\
\hline ICER & REF & Dominated & $\$ 81,591$ & $\$ 21,834$ \\
\hline \multicolumn{5}{|c|}{ Alternate cannabis $A E$ rates } \\
\hline Average QALYs & 0.476 & 0.482 & 0.486 & 0.479 \\
\hline Average cost & $\$ 6,379$ & $\$ 7,643$ & $\$ 7,167$ & $\$ 6,688$ \\
\hline ICER & REF & Dominated & $\$ 73,193$ & Ext. dominated \\
\hline \multicolumn{5}{|l|}{ Cannabis wastage } \\
\hline Average QALYs & 0.476 & 0.488 & 0.489 & 0.480 \\
\hline Average cost & $\$ 6,383$ & $\$ 7,875$ & $\$ 7,430$ & $\$ 6,797$ \\
\hline ICER & REF & Dominated & $\$ 83,865$ & Ext. dominated \\
\hline
\end{tabular}

All costs are in 2017 U.S. dollars. ICERs are calculated referent to the next least costly nondominated treatment option.

\section{Alternate time horizons}

Adjunctive cannabis remained cost-effective in analyses using time horizons of 5 and 10 years (Table 3 ). First-line adjunctive cannabis was dominated in all alternate timeframes, while third-line adjunctive cannabis was no longer extendedly dominated by secondline adjunctive cannabis, with more favorable ICERs over both 5- and 10-year time horizons.

\section{Alternate adverse event rate modifiers}

When the adverse event rate modifiers for cannabis were adjusted to reflect the exclusion of active cannabis users from the patient cohort, second-line adjunctive cannabis continued to dominate other cannabis-containing strategies (Table 3 ). The strategy produced a slightly lower increase in efficacy from base-case $(0.010$ from 0.013$)$, with a slight increase in incremental cost ( $\$ 788$ from $\$ 610)$, which resulted in an ICER of $\$ 73,193$ per QALY.

\section{Cannabis wastage}

Results of cannabis wastage subanalysis are presented in Table 3. As in base-case analysis, second-line adjunctive cannabis dominated first-line and third-line adjunctive cannabis. Applying cannabis wastage to the base-case model resulted in a significantly higher ICER $(\$ 83,865$ per QALY).

\section{Discussion}

In this exploratory CEA of smoked cannabis for neuropathic pain, we found augmentation of standard therapy agents for neuropathic pain with smoked cannabis to be cost-effective over the short- and long-term with ICERs below our designated threshold of $\$ 100,000$ per QALY. This held true when parameters were varied over their distribution ranges in probabilistic sensitivity analysis, and when more stringent inputs for adverse event rates and cannabis wastage were modeled.

First-line adjunctive cannabis was dominated by other adjunctive cannabis strategies, as exposing the entire cohort of patients to the increased risk of nonSAEs associated with cannabis use appeared to outweigh any significant gains in pain control. In contrast, when patients started cannabis after failing one or more drugs - through either repeated rounds of poor pain relief or intolerable adverse events-they derived increased utility at a more favorable cost. In the basecase analysis, this was most apparent for second-line adjunctive cannabis.

No published CEA has addressed the use of smoked cannabis, the treatment of neuropathic pain, or cannabis and cannabinoids in the United States health marketplace; our study, then, is a novel contribution to the literature in all three regards. Previous studies have evaluated the cost-effectiveness of cannabis-based medicine for the treatment of MS in the European health marketplace, with conflicting results. One combined randomized clinical trial and CEA of dronabinol to slow disease progression found that the intervention had no significant disease-modifying effect and was therefore not cost-effective. ${ }^{42}$ Four published CEAs evaluated nabiximols versus usual care for the treatment of MS-related spasticity using a willingness-topay threshold of $€ 30,000$ per QALY or $£ 30,000$ per QALY. A publicly funded study in the United Kingdom found that nabiximols were not cost-effective at that threshold, while industry-funded studies built using Spain, Germany, Italy, and Wales as health marketplace settings found nabiximols to be cost-effective. ${ }^{43-46}$

Our model was sensitive to changes affecting the quantity of cannabis purchased and, therefore, the price paid for cannabis. We overestimated quantities of cannabis consumed daily: dosing of THC was based on the weight of cannabis cigarette consumed by participants in the clinical trial by Ellis et al., ${ }^{6}$ but it was impossible to subtract the weight of the paper used to roll the cigarette. Likewise, the per-gram price figure used in their analysis may have overestimated cost, as the majority of transactions used to calculate it were subject to a $37 \%$ excise tax not levied on medicinal cannabis. ${ }^{37,47}$ In practice, patients in the United 
States pay a variety of prices, which may be much higher in some settings or significantly lower, especially if patients grow their own cannabis.

Our model incorporates parameters from a single clinical trial of good quality, a year-long observational study, and published market data to simulate the use of cannabis in clinical practice as comprehensively as possible. Nonetheless, our model has several limitations. Existing clinical trials of cannabis have been of short duration, we overestimated the dose of cannabis consumed because the weight of the paper used to roll the cigarettes was included in measurements of the dose, and the observational cohort study may under- or overestimate adverse event rates related to long-term use of cannabis.

Due to the limited scope of published clinical trials of cannabis and the absence of CEA in HIV neuropathy, we derived cannabis efficacy parameters from participants with HIV neuropathy and then applied them to a published CEA model of pDPN. However, there is evidence in the literature to suggest that this is a reasonable extrapolation: pain relief from standard therapy agents is similar in HIV neuropathy and $\mathrm{pDPN}^{48-53}$; treatment guidelines for both pDPN and HIV neuropathy recommend standard therapy agents as first- or second-line agents ${ }^{54-56}$; short-term effects of smoked cannabis for pDPN are consistent with effects observed in trials on HIV neuropathy. ${ }^{6-8,11}$

Another limitation is that there are no studies of inhaled medical cannabis examining "tolerance" to cannabis over the long-term, and tolerance was not accounted for in the model. However, a study of cannabinoids in MS suggest continued analgesic effects over time. ${ }^{57}$ Furthermore, our model inputs for adverse events were derived from an observational cohort, in which patients administered their study cannabis by multiple routes, including smoking, vaporization, and oral consumption (the majority of patients-61\%using some combination of the three ${ }^{35}$ ). As such, they may not be generalizable to an exclusively smoked cannabis regimen.

Because there are no available clinical trial data on long-term consequences of regular licit, smoked medicinal cannabis use-with data on long-term outcomes derived from studies of recreational or other illicit use $^{58}$ - we did not structure provisions for latent, insidious or long-term adverse effects into the model.

\section{Conclusion}

Notwithstanding limitations, this is the first published CEA of inhaled cannabis for any condition. The results of our analysis indicate that should long-term consequences and efficacy be similar to what has been observed in published trials, smoked medicinal cannabis is a useful tool from a cost-effectiveness perspective for the treatment of chronic neuropathic pain. Judicious use of medicinal cannabis alongside standard therapy agents may be particularly beneficial to patients with refractory pain and to active cannabis users. Our findings are concordant with clinical experience and published guidelines that recommend consideration of cannabis for patients nonresponsive to initial treatment. ${ }^{16}$

The data demonstrate the importance of cost to the ultimate utility of medicinal cannabis in practice. In addition to exploring the efficacy and safety profile of cannabis-based medicine, including cannabis or cannabinoids as monotherapy for neuropathic pain, future research should evaluate its economic feasibility and influence public policy to assure that this potentially useful intervention is accessible. Characterizing the cost-effectiveness of medicinal cannabis will inform future research and policy as to whether this treatment modality is promising from a health economics and population health perspective, over and above its emerging efficacy in clinical trials.

\section{Acknowledgments}

We are grateful to Ben Gouaux of the University of California Center for Medicinal Cannabis Research for the provision of clinical trial data. This project was partially supported by the National Institutes of Health grant 1TL1TR001443 of CTSA funding. The content is solely the responsibility of the authors and does not necessarily represent the official views of the NIH.

\section{Author Disclosure Statement}

No competing financial interests exist.

\section{References}

1. Rog DJ, Nurmikko TJ, Friede T, et al. Randomized, controlled trial of cannabis-based medicine in central pain in multiple sclerosis. Neurology. 2005;65:812-819.

2. Nurmikko TJ, Serpell MG, Hoggart B, et al. Sativex successfully treats neuropathic pain characterised by allodynia: a randomised, double-blind, placebo-controlled clinical trial. Pain. 2007;133:210-220.

3. Svendsen KB, Jensen TS, Bach FW. Does the cannabinoid dronabinol reduce central pain in multiple sclerosis? Randomised double blind placebo controlled crossover trial. BMJ. 2004;329:253.

4. Frank B, Serpell MG, Hughes J, et al. Comparison of analgesic effects and patient tolerability of nabilone and dihydrocodeine for chronic neuropathic pain: randomised, crossover, double blind study. BMJ. 2008;336: 199-201.

5. Toth C, Mawani S, Brady S, et al. An enriched-enrolment, randomized withdrawal, flexible-dose, double-blind, placebo-controlled, parallel assignment efficacy study of nabilone as adjuvant in the treatment of diabetic peripheral neuropathic pain. Pain. 2012;153:2073-2082. 
6. Ellis RJ, Toperoff W, Vaida F, et al. Smoked medicinal cannabis for neuropathic pain in HIV: a randomized, crossover clinical trial. Neuropsychopharmacology. 2009;34:672-680.

7. Abrams DI, Jay CA, Shade SB, et al. Cannabis in painful HIV-associated sensory neuropathy. Neurology. 2007;68:515-521.

8. Ware MA, Wang T, Shapiro S, et al. Smoked cannabis for chronic neuropathic pain: a randomized controlled trial. CMAJ. 2010;182:E694-E701.

9. Wallace MS, Marcotte TD, Umlauf A, et al. Efficacy of inhaled cannabis on painful diabetic neuropathy. J Pain. 2015;16:616-627.

10. Wilsey $B$, Marcotte $T$, Deutsch $R$, et al. Low-dose vaporized cannabis significantly improves neuropathic pain. J Pain. 2013;14:136-148.

11. Wilsey B, Marcotte T, Tsodikov A, et al. A randomized, placebo-controlled, crossover trial of cannabis cigarettes in neuropathic pain. J Pain. 2008;9: 506-521.

12. Sadosky A, Mardekian J, Parsons B, et al. Healthcare utilization and costs in diabetes relative to the clinical spectrum of painful diabetic peripheral neuropathy. J Diabetes Complications. 2015;29:212-217.

13. Gore M, Brandenburg NA, Dukes $E$, et al. Pain severity in diabetic peripheral neuropathy is associated with patient functioning, symptom levels of anxiety and depression, and sleep. J Pain Symptom Manage. 2005;30:374-385.

14. Doth $A H$, Hansson PT, Jensen MP, et al. The burden of neuropathic pain: a systematic review and meta-analysis of health utilities. Pain. 2010;149: 338-344.

15. Andreae $\mathrm{MH}$, Carter GM, Shaparin N, et al. Inhaled cannabis for chronic neuropathic pain: a meta-analysis of individual patient data. J Pain. 2015; 16:1221-1232.

16. Moulin D, Boulanger A, Clark AJ, et al. Pharmacological management of chronic neuropathic pain: revised consensus statement from the Canadian Pain Society. Pain Res Manag. 2014;19:328-335.

17. Hasin DS, Saha TD, Kerridge BT, et al. Prevalence of marijuana use disorders in the United States between 2001-2002 and 2012-2013. JAMA Psychiatry. 2015;72:1235-1242.

18. Park JY, Wu LT. Prevalence, reasons, perceived effects, and correlates of medical marijuana use: a review. Drug Alcohol Depend. 2017;177:1-13.

19. Ryan-Ibarra S, Induni M, Ewing D. Prevalence of medical marijuana use in California, 2012. Drug Alcohol Rev. 2015;34:141-146.

20. Chong MS, Wolff $K$, Wise $K$, et al. Cannabis use in patients with multiple sclerosis. Mult Scler. 2006;12:646-651.

21. Reinarman $C$, Nunberg $H$, Lanthier $F$, et al. Who are medical marijuana patients? Population characteristics from nine California assessment clinics. J Psychoact Drugs. 2011;43:128-135.

22. Nunberg $\mathrm{H}$, Kilmer B, Pacula RL, et al. An analysis of applicants presenting to a medical marijuana specialty practice in California. J Drug Policy Anal. 2011;4:pii: 1.

23. Ilgen MA, Bohnert $K$, Kleinberg F, et al. Characteristics of adults seeking medical marijuana certification. Drug Alcohol Depend. 2013;132:654-659.

24. Sanders GD, Neumann PJ, Basu A, et al. Recommendations for conduct, methodological practices, and reporting of cost-effectiveness analyses. JAMA. 2016;316:1093-1103.

25. Torrance GW. Measurement of health state utilities for economic appraisal. J Health Econ. 1986;5:1-30.

26. Neumann PJ, Cohen JT, Weinstein MC. Updating cost-effectiveness-the curious resilience of the $\$ 50,000$-per-QALY threshold. N Engl J Med. 2014; 371:796-797.

27. Braithwaite RS, Meltzer DO, King JT, et al. What does the value of modern medicine say about the $\$ 50,000$ per quality-adjusted life-year decision rule? Med Care. 2008;46:349-356.

28. Grosse SD. Assessing cost-effectiveness in healthcare: history of the $\$ 50,000$ per QALY threshold. Expert Rev Pharmacoecon Outcomes Res. 2008;8:165-178.

29. Herzog S, Shanahan M, Grimison P, et al. Systematic review of the costs and benefits of prescribed cannabis-based medicines for the management of chronic illness: lessons from multiple sclerosis. Pharmacoeconomics. 2018;36:67-78.

30. Angevine PD, Berven S. Health economic studies: an introduction to costbenefit, cost-effectiveness, and cost-utility analyses. Spine (Phila Pa 1976). 2014;39(22 Suppl 1):S9-S15.

31. Bellows BK, Nelson RE, Oderda GM, et al. Long-term cost-effectiveness of initiating treatment for painful diabetic neuropathy with pregabalin, duloxetine, gabapentin, or desipramine. Pain. 2016;157:203-213.
32. Gordon J, Lister S, Prettyjohns M, et al. A cost-utility study of the use of pregabalin in treatment-refractory neuropathic pain. J Med Econ. 2012; 15:207-218.

33. Wu EQ, Birnbaum HG, Mareva MN, et al. Cost-effectiveness of duloxetine versus routine treatment for U.S. patients with diabetic peripheral neuropathic pain. J Pain. 2006;7:399-407.

34. Annemans L, Caekelbergh K, Morlion B, et al. A cost-utility analysis of pregabalin in the management of peripheral neuropathic pain. Acta Clin Belg. 2008;63:170-178.

35. Ware MA, Wang T, Shapiro $S$, et al. Cannabis for the management of pain: assessment of safety study (COMPASS). J Pain. 2015;16:1233-1242.

36. Coleman $\mathrm{Cl}$, Limone B, Sobieraj DM, et al. Dosing frequency and medication adherence in chronic disease. J Manag Care Pharm. 2012;18:527-539.

37. Smart R, Caulkins JP, Kilmer B, et al. Variation in cannabis potency and prices in a newly legal market: evidence from 30 million cannabis sales in Washington state. Addiction. 2017;112:2167-2177.

38. Sifaneck SJ, Ream GL, Johnson BD, et al. Retail marijuana purchases in designer and commercial markets in New York City: sales units, weights, and prices per gram. Drug Alcohol Depend. 2007;90:S40-S51.

39. Clements KW. Three facts about marijuana prices. Aust J Agric Res Econ. 2004;48:271-300.

40. Average price per ounce of high quality marijuana as of November 2017, by selected U.S. state (in U.S. dollars). Statistia. Available at: www.statista.com (last accessed on December 6, 2017).

41. Jones RT, Benowitz NL, Herning RI. Clinical relevance of cannabis tolerance and dependence. J Clin Pharmacol. 1981;21:143S-152S.

42. Ball S, Vickery J, Hobart J, et al. The cannabinoid use in progressive inflammatory brain disease (CUPID) trial: a randomised double-blind placebo-controlled parallel-group multicentre trial and economic evaluation of cannabinoids to slow progression in multiple sclerosis. Health Technol Assess. 2015;19:vii-viii, xxv-xxxi, 1-187.

43. Lu L, Pearce $H$, Roome $C$, et al. Cost effectiveness of oromucosal cannabisbased medicine (Sativex ${ }^{\mathbb{R}}$ ) for spasticity in multiple sclerosis. Pharmacoeconomics. 2012;30:1157-1171.

44. Slof J, Gras A. Sativex ${ }^{\circledR}$ in multiple sclerosis spasticity: a cost-effectiveness model. Expert Rev Pharmacoecon Outcomes Res. 2012;12:439-441.

45. Slof J, Ruiz L, Vila C. Cost-effectiveness of Sativex in multiple sclerosis spasticity: new data and application to Italy. Expert Rev Pharmacoecon Outcomes Res. 2015;15:379-391.

46. Gras A, Broughton J. A cost-effectiveness model for the use of a cannabisderived oromucosal spray for the treatment of spasticity in multiple sclerosis. Expert Rev Pharmacoecon Outcomes Res. 2016;16:771-779.

47. Taxes due on Marijuana. Washington State Department of Revenue. Available at: https://dor.wa.gov (last accessed on February 5, 2018).

48. Hahn K, Arendt G, Braun JS, et al. A placebo-controlled trial of gabapentin for painful HIV-associated sensory neuropathies. J Neurol. 2004;251: 1260-1266.

49. Kieburtz K, Simpson D, Yiannoutsos C, et al. A randomized trial of amitriptyline and mexiletine for painful neuropathy in HIV infection. Neurology. 1998;51:1682-1688.

50. Dinat N, Marinda E, Moch S, et al. Randomized, double-blind, crossover trial of amitriptyline for analgesia in painful hiv-associated sensory neuropathy. PLoS One. 2015;2:10:e0126297.

51. Shlay JC, Chaloner K, Max MB, et al. Acupuncture and amitriptyline for pain due to hiv-related peripheral neuropathy. JAMA. 1998;280:1590-1595.

52. Simpson DM, Schifitto G, Clifford DB, et al. Pregabalin for painful HIV neuropathy a randomized, double-blind, placebo-controlled trial. Neurology. 2010;74:413-420.

53. Simpson DM, Rice AS, Emir B, et al. A randomized, double-blind, placebocontrolled trial and open-label extension study to evaluate the efficacy and safety of pregabalin in the treatment of neuropathic pain associated with human immunodeficiency virus neuropathy. Pain. 2014;155:1943-1954.

54. Snyder MJ, Gibbs LM, Lindsay TJ. Treating painful diabetic peripheral neuropathy: an update. Am Fam Phys. 2016;94:227-234.

55. Bruce RD, Merlin J, Lum PJ, et al. 2017 HIV medicine association of infectious diseases society of America clinical practice guideline for the management of chronic pain in patients living with human immunodeficiency virus. Clin Infect Dis. 2017;65:1601-1606.

56. Pop-Busui R, Boulton AJ, Feldman EL, et al. Diabetic neuropathy: a position statement by the American diabetes association. Diabetes Care. 2017;40:136-154. 
57. Zajicek JP, Sanders HP, Wright DE, et al. Cannabinoids in multiple sclerosis (CAMS) study: safety and efficacy data for 12 months follow up. J Neurol Neurosurg Psychiatry. 2005;76:1664-1669.

58. Hall W, Degenhardt L. Adverse health effects of non-medical cannabis use. Lancet. 2009;374:1383-1391.

59. HCUPnet-Hospital Inpatient National Statistics. Healthcare cost and utilization project. Available at: https://hcupnet.ahrq.gov (last accessed on February 5, 2018).

60. Oladapo AO, Barner JC, Rascati KL, et al. A retrospective database analysis of neuropathic pain and oral antidiabetic medication use and adherence among Texas adults with type 2 diabetes enrolled in Medicaid. Clin Ther. 2012;34:605-613.

61. Chen HW, Bercik RS, Werner EF, et al. Cost-effectiveness of percutaneous tibial nerve stimulation versus extended release tolterodine for overactive bladder. J Urol. 2012;187:178-184.

62. Wu JM, Fulton RG, Amundsen $\mathrm{CL}$, et al. Patient preferences for different severities of and treatments for overactive bladder. Female Pelvic Med Reconstr Surg. 2011;17:184-189.

63. Smith C, Crowther C, Beilby J, et al. The impact of nausea and vomiting on women: a burden of early pregnancy. Aust N Z J Obstet Gynaecol. 2000; 40:397-401.

64. Wielage RC, Bansal M, Andrews JS, et al. The cost-effectiveness of duloxetine in chronic low back pain: a US private payer perspective. Value Health. 2013;16:334-344.

65. Greiner W, Lehmann K, Earnshaw S, et al. Economic evaluation of durogesic in moderate to severe, nonmalignant, chronic pain in Germany. Eur J Health Econ. 2006;7:290-296.

66. National Collaborating Centre for Chronic Conditions. Appendix D: Details of the NSAID/COX-2 inhibitor health economic model. Osteoarthritis: National Clinical Guideline for Care and Management in Adults. London: Royal College of Physicians, 2008.

67. Tsevat J, Goldman L, Soukup JR, et al. Stability of time-tradeoff utilities in survivors of myocardial infarction. Med Decis Mak. 1993;13:161165.

68. CMS. Physician fee schedule. Centers for Medicare \& Medicaid Services. Available at: https://www.cms.gov/apps/physician-fee-schedule/ (last accessed on February 5, 2018).

69. Medical Expenditure Panel Survey (MEPS). Outpatient visits, 2015. Agency for Healthcare Research and Quality. Available at: www.ahrq.gov/data/ meps.html (last accessed on March 23, 2018).
70. Medical Expenditure Panel Survey (MEPS). Hospital inpatient stays, 2015. Agency for Healthcare Research and Quality. Available at: www.ahrq.gov/data/meps.html (last accessed on March 23, 2018).

71. Consumer Price Index Inflation Calculator. Bureau of Labor Statistics. Available at: https://data.bls.gov/cgi-bin/cpicalc.pl (last accessed on February 5, 2018).

72. Medical Expenditure Panel Survey (MEPS). Prescribed medicines, 2015. Agency for Healthcare Research and Quality. Available at: www.ahrq.gov/ data/meps.html (last accessed on March 23, 2018).

Cite this article as: Tyree GA, Sarkar R, Bellows BK, Ellis RJ, Atkinson JH, Marcotte TD, Wallace MS, Grant I, Shi Y, Murphy JD, Grelotti DJ (2019) A cost-effectiveness model for adjunctive smoked cannabis in the treatment of chronic neuropathic pain, Cannabis and Cannabinoid Research 4:1, 62-72, DOI: 10.1089/can.2018.0027.

Abbreviations Used
$\mathrm{AE}=$ adverse event
COMPASS $=$ Cannabis for the Management of Pain: Assessment
$\quad$ of Safety Study
$\mathrm{Cl}=$ confidence interval
$\mathrm{CEA}=$ cost-effectiveness analysis
$\mathrm{ICER}=$ incremental cost-effectiveness ratio
$\mathrm{MS}=$ multiple sclerosis
$\mathrm{ORs}=$ odds ratios
$\mathrm{pDPN}=$ painful diabetic peripheral neuropathy
$\mathrm{QALYs}=$ quality-adjusted life years
$\mathrm{REF}=$ reference value
$\mathrm{SD}=$ standard deviation
$\mathrm{SE}=$ standard error
$\mathrm{SAEs}=$ serious adverse events
$\mathrm{THC}=$ tetrahydrocannabinol

